\title{
Impact of bed surface arrangement on bedload rate: comparisons between loose, armored and water-worked beds
}

\author{
Emeline Perret ${ }^{1}$, Céline Berni ${ }^{1}$, and Benoît Camenen ${ }^{1}$ \\ ${ }^{1}$ Irstea, UR RiverLy, centre de Lyon-Villeurbanne, 5 rue de la Doua CS 20244, 69625 Villeurbanne, \\ France
}

\begin{abstract}
Existing formulas for predicting bedload rate may be not adapted for mountain rivers with poorly sorted sediments, partly because they were often established using laboratory data with conditions far from those found in such rivers. Natural bed arrangement is particularly difficult to reproduce in flumes, although recent studies highlighted its importance on bedload dynamics. This study aims to quantify bed arrangement impact on bedload rate using original laboratory tests and to improve existing bedload formulas. Three types of bed composed with the same material but having different bed arrangements were studied: loose beds were installed manually in the flume and the others, packed and water-worked beds, were created using water power. Packed beds were assimilated to flat beds composed of a static armor layer whereas water-worked beds exhibited stronger bed organization, including large-scale bed forms. Laser-scanner surveys were used to characterize differences in bed morphology. Similar unsteady hydraulic conditions were applied over these beds. Results showed that bedload dynamics varies significantly depending on the initial arrangement. Compared to loose bed, bedload was enhanced over waterworked bed and reduced over packed bed. Bed surface indicators are thus important parameters to take into account when predicting gravel transport.
\end{abstract}

\section{Introduction}

Bedload rate estimation in mountain rivers is crucial since these rivers are generally constrained and subjected to many hazard and vulnerability, which impacts directly their morphological bed evolutions. Existing bedload formulas often fail to reproduce the bedload dynamics of such rivers where sediments are poorly sorted. Most of them were established in laboratory for well-sorted sediments, and do not take into account aspects such as the bed material grain size distribution or the role played by bed surface structures and forms, namely the bed arrangement. Few laboratory studies highlighted nevertheless the importance of considering bed arrangement. It has been observed that during constant hydraulics conditions a gravel-bed reorganizes itself, inducing change in bedload rates (decline) [1, 2]. Bed surface coarsening [2], increase in grain imbrication [3], and formation of grain clusters [4] are the main observed phenomena that could influence bedload. Some hysteresis bedload patterns were also observed in unsteady flows experiments $[5,6]$, reflecting changes in bed surface organization. All these studies agree that antecedent flow 
history and initial degree of bed armouring are determinant for bedload rate prediction. These observations were consistent with in-situ measurements [7, 8], where bedload rates were found different even with similar hydraulic conditions. A large and short flood event can indeed be powerful enough to break bed patterns, leading to an increase in bedload rate.

The first aim of this study is to highlight the importance of considering bed arrangement when predicting bedload rate. Although previous studies had identified bed arrangement as an important factor affecting gravel mobility, to our knowledge, no study tried to precisely link it to bedload dynamics. We try to fill this gap by studying the bedload dynamics of beds having different types of arrangement. Precise topographical measurements of the beds were made to determine indicators describing their surface arrangement and to correlate them with bedload rates. Published bedload formulas were also tested to know their limitations and to suggest new improvements. The ultimate aim of this study is to provide recommendations concerning bed surface parameters that need to be integrated in a future bedload formula to better approach the dynamics observed in rivers.

\section{Experimental set-up}

\subsection{Materials}

The tests were performed using the HHLab tilting flume located at Irstea LyonVilleurbanne $(18 \mathrm{~m} \times 1 \mathrm{~m} \times 0.85 \mathrm{~m})$. A feeding system was installed at the upstream end of the flume to feed homogeneously and continuously the channel with sediments. A 1m-long adjustable weir was installed at the downstream end of the flume to control the water depth $(h)$. Measurement devices were set on a mobile platform, which moves in all directions: longitudinal $(x)$, transverse $(y)$, and perpendicular to the bottom $(z)$. The origin of the axes was located at the right side of the flume's upstream end, at the bottom channel. The water depth and bed topography were measured using Ultrasonic sensors (Microsonic mic+130/IU/TC) and a laser-scanner (scanControl 2900 Micro-Epsilon), respectively. The beds were composed of moderately sorted gravel $(\mathrm{G})$, which were angular with a median diameter $D_{50}=6.8 \mathrm{~mm}$, a geometric standard deviation of 1.4 and a relative density of 2.65.

\subsection{Sediment transport experiments}

Using the results of sediment transport runs, the gravel dynamics of beds having diverse arrangements were studied. There was no upstream gravel feeding during the tests. These experiments consist in operating the flume with a stepped flow hydrograph while collecting transported gravel periodically at the flume's downstream end. Each test was denoted with the type of bed studied (L-G, P-G and WW-G for loose, packed, and water-worked gravel beds, respectively) completed by an test number $i$. Note that experiments sharing the same $i$ refer to runs performed successively with no manual gravel bed reinstallation (e.g L-G-1 followed by P-G-1).

The experimental configurations were chosen to represent mountain rivers (channel slope was set to $1 \%$ for all tests, Shields numbers $\left(\tau^{*}\right)$ and the relative roughness $\left(D_{50} / h\right)$ varied between $0.04-0.08$ and $0.08-0.14$, respectively). The stepped hydrographs simulated unsteady flow events. They were similar in terms of magnitude, duration and sequencing for all tests, namely characterized by symmetrical rising and falling limbs with steady and uniform flow intervals and transitions. We chose to apply discontinuous hydrographs for practical reasons. Constant plateaus were necessary to carry out a large number of measurements in a minimum of time. The plateaus remained nevertheless shorter than the characteristic time for bed arrangement (several hours) [1,9]. 
Before starting the experiment, the bed surface was surveyed using the laser-scanner. During the test, transported gravels were collected manually every 5 minutes at the flume's downstream end to measure bedload rate per unit of width $\left(q_{s}\right)$. $h$ was measured during each plateau along the flume centerline. At the end, the bed was drained and a new bed surface topography was measured. For details about the protocol, please refer to [10].

\subsection{Bed set-up}

A particular attention was paid to the bed creation, which was conducted to obtain naturelike beds with diverse arrangements. To create loose beds (L-G), gravel was manually installed in the flume as it was commonly done in previous studies. A scraper was used to obtain an $8 \mathrm{~cm}$-thick flatbed surface, parallel to the flume bottom. L-G beds denote nonorganized beds that were not subjected to antecedent flows. They are not representative of natural rivers, but they were taken as a reference to compare with previous studies.

Antecedent long flows were necessary to form a packed bed (P-G). Its formation followed three steps. First, a L-G bed was installed in the flume. Second, a sediment transport experiment was performed on this bed. Third, the bed was subjected to a constant flow chosen to be equal to the flow necessary for putting into motion gravel grains. This lasted approximately 12 hours (i.e. the time necessary for $q_{s}$ to become insignificant). P-G beds refer to organized beds. Their conditions of formation approached those for the creation of a static armor in natural flow, meaning extended periods of low flows with reduced sediment supply. In that case, the flow produced low bed shear stresses that entrained only fine or unstable grains present on the bed surface. This led to a coarser bed surface, an increase in grain imbrications and in grain patterns such as clusters [2,5].

Water-worked beds (WW-G) were created only by water power, following the methodology proposed in [11]. The method consists in feeding sediments into a running flume to create the bed. Consequently, the subsurface and surface of a WW-G bed exhibit strong organization and complexity, close to those found in the field. We tried to form a water-worked bed, which had approximately the same thickness as L-G and P-G beds to facilitate the comparison of bedload dynamics between tests. Its creation is a long process, since around 2.3 tons of grains were needed to form an $8 \mathrm{~cm}$-thick bed. We estimated specific hydraulic conditions and feeding rates in order to create it in a limited time (2 days). We set the channel slope at $1.8 \%$ and the flow discharge at $45 \mathrm{~L} / \mathrm{s}$. These conditions induced a flow transport capacity of $25 \mathrm{~g} / \mathrm{s}$ according to results observed in a run performed on a loose bed. The feeding rate was adjusted to two or three times the flow transport capacity to ensure gravel depositions during the creation. In the middle of the set-up, the channel slope was reset at $1.3 \%$ because no more deposition at $1.8 \%$ was observed. We considered the WW-G bed formed when $q_{s}$ at the entrance equals $q_{s}$ at the exit of the flume (i.e. when bed morphology becomes stable).

\section{Results and discussions}

\subsection{Bedload dynamics over the different beds}

Figure 1 shows the typical temporal variations of flow and bedload rates for tests performed on L-G, P-G and WW-G beds. All bedload evolutions followed a single-peak shape with $q_{s}$ differing according to the type of bed and varying between the hydrograph's rising and falling limb. $q_{s}$ during WW-G-12 reach values up to $20 \mathrm{~g} / \mathrm{m} / \mathrm{s}$, which are higher than those obtained during L-G or P-G bed runs. 


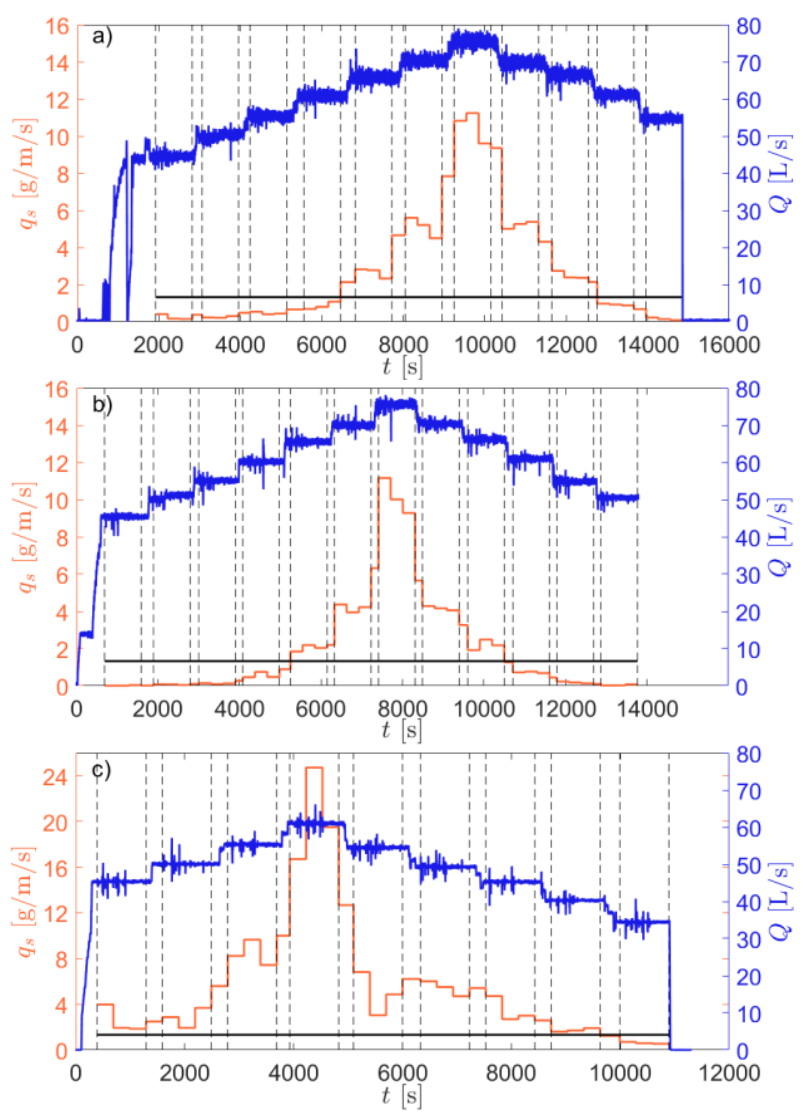

Fig. 1. Time variations of $q_{s}$ and $Q$ during tests performed on (a) a loose (L-G-3), (b) a packed (P-G3 ) or (c) a water-worked (WW-G-12) bed. Grid delimits steady steps from transitions zones.

Figure 2 compares the relationship between dimensionless bedload rate $q_{s}{ }^{*}=q_{s} /[(s-$ 1) $\left.\left.g D_{50}{ }^{3}\right]^{1 / 2}\right)$ and dimensionless bed shear stress $\tau^{*}=\tau /\left(\rho_{s}-\rho\right) g D_{50}$ for all tests $\left(\tau=\rho g R_{\mathrm{h}} J\right.$ : bed shear stress, $s=\rho_{s} / \rho$ : relative grain density, $\rho_{s}$ and $\rho$ : sediment and water density, respectively, $g$ : gravitational acceleration, $R_{\mathrm{h}}$ : hydraulic radius, $J$ : energy slope equal to bed slope for uniform flow). Data collected during rising and falling limbs were separated to highlight potential hysteresis patterns.

For a given $q_{s}{ }^{*}, \tau^{*}$ was always lower for a WW-G bed than for L-G beds and globally lower for L-G beds than for P-G beds during the rising limb. On the contrary, during the falling limb, the relationships $\left(\tau^{*}, q_{s}{ }^{*}\right)$ for L-G and P-G bed became similar. The hydrograph seems to reset previous shear stress histories, so that L-G and P-G beds present same bedload dynamics and similar bed surface arrangements (see data referring to the final bed state in Table 1). However, the relationships $\left(\tau^{*}, q_{s}{ }^{*}\right)$ did not change for WW-G bed.

Clockwise bedload hysteresis patterns were observed for L-G bed experiments, meaning that $q_{s}$ were larger during the rising limb than during the falling limb for same shear stresses. The beds organized during the rising limb leading to a stronger bed surface arrangement during the falling limb. For P-G bed tests, counter-clockwise hysteresis patterns may be noticed, meaning that $q_{s}$ were smaller during the rising limb than during the falling limb for same shear stresses. Bed structures present during the rising limb were probably broken inducing weaker bed surface organization during the falling limb and enhancing $q_{s}$. A slight clockwise pattern was detected for WW-G bed, which is probably due to changes in bed surface organization during the falling limb (see Section 3.2). 


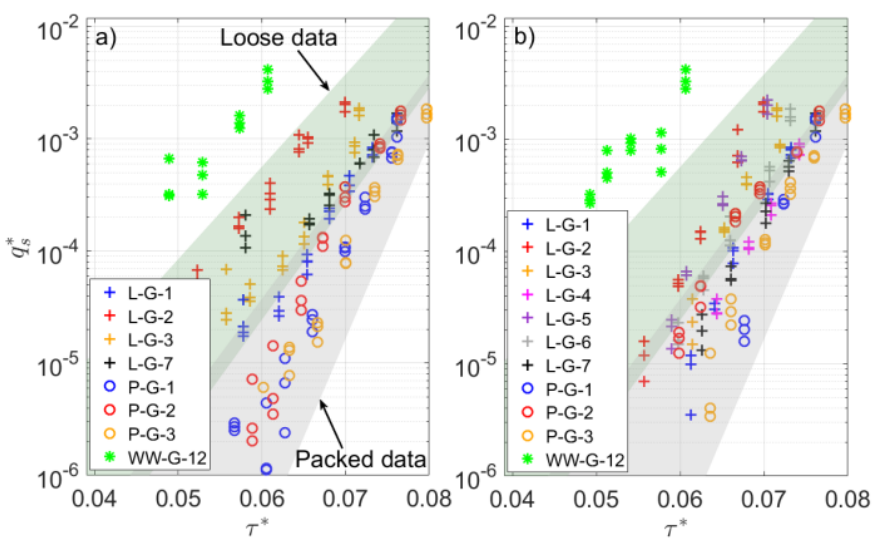

Fig. 2. Evolution of the dimensionless bedload rate according to the dimensionless bed shear stress for all tests (a) at the rising and (b) falling limb of the hydrograph.

We expected that all data would collapse during the rising limb for one type of bed since the same protocol was applied. However, differences are distinguishable; particularly for data from L-G bed runs. Measurement uncertainty alone cannot explain the differences. The results raise questions about experiment reproducibility. The scatter could be explained by differences in initial bed arrangements that would impact bedload dynamics. The analysis of the laser-scanner data confirms this assumption (Table 1). For P-G beds, data are more regrouped suggesting a better reproducibility when using water power. Despite the differences, data from L-G bed tests are grouped together in the green area of the $\left(\tau^{*}, q_{s}{ }^{*}\right)$ plot. Data from P-G bed tests grouped in another area involving higher $\tau^{*}$. The intersection of these areas provided a location for slightly packed beds. Data from WW-G bed were located outside these two areas in a zone of high $q_{s}{ }^{*}$ related to smaller $\tau^{*}$. At the falling limb, the water-worked bed arrangement was still different from the others explaining why WW-G bed data stay in the same area as the one observed during the rising limb (Table 1).

\subsection{Influence of bed arrangement}

Our results showed that even small changes on the surface can induce strong variations in terms of $q_{s}$. To investigate the bed arrangement influence, it is necessary to quantify the changes in bed morphology between the different beds. To do that, we analysed the laserscanner data (see [12]). Figure 3c shows the entire topography of WW-G bed. The range of bed elevations was higher than for the other beds, which were quite flat (Figure 3). Bed arrangement of WW-G bed was complex with presence of large bedforms (bars). Characteristics of the smallest and largest macroforms were reported in Table $1\left(\Delta_{x 0}, \sigma_{z l}\right.$ and $\Delta_{y 0}, \sigma_{z t}$ are the bedform length and roughness in the stream-wise direction and the bedform length and roughness in cross-stream directions, respectively). The bed elevation analysis at large scale showed that bed slope of the WW-G bed was in average lower than slopes for the other beds, which should lead to bedload reduction. However, the opposite behavior was noted (Figure 2). This was partly explained by presence of bars, which created pathways where flow was accelerated enhancing gravel transport. During the falling limb, bars were smoothed and disappeared progressively, which reduced $q_{s}$ (Figure $3 \mathrm{~d}$ ).

In general, the geometrical grain roughness $\sigma_{g}$ was smaller for beds formed with water power compared to L-G bed (Table 1). This revealed a better grain imbrication and thus a smoother bed surface. $q_{s}$ should be reduced in these cases. This assessment was verified for P-G bed tests and not for WW-G bed run. The impact of macro-bedforms seems thus more important on $q_{s}$ than a change in bed stability. The initial armoring degree $S_{k}$ was more 
pronounced for P-G beds $\left(S_{k}>0\right)$ than for L-G and WW-G beds, which again revealed a stronger bed stability. For L-G and P-G beds, no large bedform was noticed. However, micro-forms (structures) were present in P-G beds. Their lengths $\left(\Delta_{x 0}, \Delta_{y 0}\right)$ at the beginning of the experiment did not exceed $2 \times D_{50}$, but they also contributed to increase bed stability. Gravel was randomly organized in L-G beds, whereas in P-G beds it was orientated in the streamwise direction ( $\Phi$ close to 0 and $a / b>1$, where $\Phi$ is the angle between grain and flow direction and $a / b$ refers to the phenomena intensity). The drag force for gravel orientated in streamwise direction is in average smaller than for randomly orientated gravel, which reduces $q_{s}$. Regarding the final indicators, it can be noted that L-G beds experienced more reorganization at the grain scale than the P-G or WW-G beds, which leads to a significant difference in $q_{s}$ between the rising and falling limbs. The analysis of one indicator cannot explain bedload evolution but a combination of these latter might. Our data were not sufficient to find a relationship relating $q_{s}$ and bed arrangement criteria. Some correlations were though highlighted. $q_{s}$ decreases with decreasing $\sigma_{g}$, with presence of bed structures and preferential grain orientation, and with increasing bed armoring degree. $q_{s}$ increases with presence of large bedforms that create significant flow pathways.
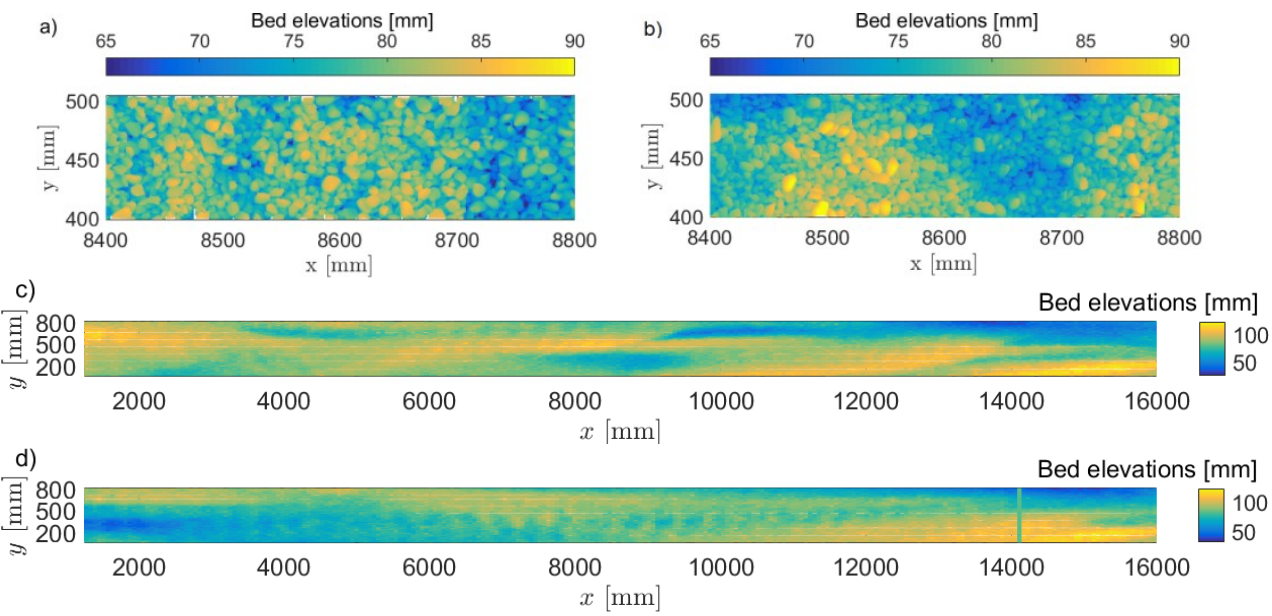

Fig. 3. Zoom on bed surface topography of (a) a loose (L-G-3) and (b) a packed (P-G-3) bed before starting the tests. Bed topography of a water-worked bed (WW-G-12) (c) before and (d) after a test.

Table 1. Bed surface indicators deduced from the laser scanner surveys. $\Delta_{x 0}$ and $\Delta_{y 0}$ were estimated as in [12] for L-G and P-G bed data and by eyes for WW-G bed data (Fig.3c). Bold values refer to the initial bed state in contrast to the others which refer to the final bed state.

\begin{tabular}{|c|c|c|c|c|c|c|c|c|c|c|c|c|c|c|c|c|}
\hline Exp. & \multicolumn{2}{|c|}{$\sigma_{g}[\mathrm{~mm}]$} & \multicolumn{2}{|c|}{$S_{k}[-]$} & \multicolumn{2}{|c|}{$\sigma_{z l}[\mathrm{~mm}]$} & \multicolumn{2}{|c|}{$\sigma_{z t}[\mathrm{~mm}]$} & \multicolumn{2}{|c|}{$\boldsymbol{\Delta}_{x \boldsymbol{0}}[\mathrm{mm}]$} & \multicolumn{2}{|c|}{$\Delta_{v,}[\mathrm{~mm}]$} & \multicolumn{2}{|c|}{$a / b[-]$} & \multicolumn{2}{|c|}{$\boldsymbol{\Phi}\left[{ }^{\circ}\right]$} \\
\hline L-G-1 & 3.9 & 3.4 & -0.28 & 0.02 & 4.3 & 4.2 & 4.2 & 4.3 & 7.9 & 7.9 & 7.3 & 7.3 & 1.0 & 1.1 & 18.1 & -9 \\
\hline L-G-2 & 3.2 & 3.5 & -0.34 & 0.14 & 3.5 & 4.3 & 3.2 & 4.2 & 6.8 & 10.6 & 6.6 & 8.8 & 1.1 & 1.3 & 22.4 & 6.2 \\
\hline L-G-3 & 3.7 & 3.4 & -0.35 & 0.02 & 4.1 & 5.8 & 4.1 & 4.6 & 6.9 & 8.8 & 6.8 & 8.4 & 1.1 & 1.2 & -20.5 & 1.0 \\
\hline L-G-4 & 3.7 & 3.8 & -0.49 & 0.09 & 4.2 & 4.1 & 3.9 & 4.6 & 7.1 & 10.9 & 6.8 & 9.6 & 1.1 & 1.3 & -21.5 & 4.7 \\
\hline L-G-5 & 3.8 & 3.8 & -0.48 & 0.01 & 4.4 & 3.6 & 4.0 & 4.1 & 7.3 & 10.7 & 7.2 & 9.6 & 1.1 & 1.2 & -5.0 & 1.1 \\
\hline L-G-6 & 3.9 & 3.7 & -0.41 & 0.08 & 4.3 & 4.1 & 3.8 & 3.1 & 7.7 & 11.5 & 7.1 & 10 & 1.1 & 1.3 & 10.1 & -5.3 \\
\hline L-G-7 & 3.8 & 3.5 & $\begin{array}{c}-0.40 \\
\end{array}$ & 0.09 & 4.3 & 3.9 & 3.9 & 3.9 & 7.2 & 9.9 & $\begin{array}{l}6.8 \\
\end{array}$ & 9.1 & 1.1 & 1.1 & -7.4 & 2.6 \\
\hline P-G-1 & 3.3 & 3.6 & 0.00 & -.04 & 4.6 & 6.1 & 4.3 & 4.5 & 8.3 & 10 & 7.3 & 9.0 & 1.2 & 1.2 & -6.2 & 12.3 \\
\hline P-G-2 & 3.7 & 4.0 & 0.28 & 0.16 & 5.0 & 6.5 & 4.2 & 4.8 & 12.1 & 14.5 & 10.4 & 12 & 1.5 & 1.4 & -4.4 & 4.3 \\
\hline P-G-3 & 3.5 & 3.3 & 0.08 & 0.06 & 5.1 & 5.7 & 4.4 & 1 & 11.3 & 9.8 & 9.8 & 9.0 & 1.4 & 1.3 & 0.8 & -1.0 \\
\hline $\begin{array}{l}\text { WW- } \\
\text { G-12 }\end{array}$ & 2.5 & 2.6 & 0.00 & 0.19 & $\begin{array}{c}7.1 \\
\text { to } \\
18\end{array}$ & $\begin{array}{c}2.1 \\
\text { to } \\
17\end{array}$ & $\begin{array}{c}3.9 \\
\text { to } \\
25\end{array}$ & $\begin{array}{l}1.4 \\
\text { to } \\
25\end{array}$ & $\begin{array}{c}2200 \\
\text { to } \\
4800\end{array}$ & $\begin{array}{c}500 \\
\text { to } \\
4800\end{array}$ & $\begin{array}{c}300 \\
\text { to } \\
500\end{array}$ & $\begin{array}{c}100 \\
\text { to } \\
400\end{array}$ & I & I & I & I \\
\hline
\end{tabular}




\subsection{Toward a new bedload formula}

Most of the existing formulas for predicting bedload do not take into account the link between bedload dynamics and bed arrangement. To once again expose its importance, we investigated the Meyer-Peter and Müller (1948), MPM, [13] and Recking (2010) [14] models. Figure 4 compared our data with the two models. Several values of dimensionless critical bed shear stress $\tau_{c r}{ }^{*}$ characterizing the incipient motion of gravel were tested to approach at best our results. Whatever the value of $\tau_{c r}{ }^{*}$ was, the MPM model failed to reproduce bedload dynamics of our tests at low bed shear stresses. The Recking model, which separates a state representing low $q_{s}$ and a state representing high $q_{s}$, provides a much more reliable estimate than MPM model. Whatever the value of $\tau_{c r}{ }^{*}$ was, the data from P-G bed tests were not well fitted. On the contrary, the model seems to be appropriate for data from L-G bed tests (with $\tau_{c r}{ }^{*}=0.06$ ). Unfortunately, these L-G bed tests were the least representative of natural river dynamics. The model also fits well the WW-G bed results with values of $\tau_{c r}{ }^{*}$ close to those generally used in the field (between 0.04 and 0.047). The Recking formula was partly developed using data measured in natural rivers, which explains its consistency to reproduce the WW-G bed data. Nevertheless, all the cited models failed to represent static armored beds. They were not able to differentiate the different bedload dynamics. Thus, we suggest that new formulas should take into account bed arrangement parameters previously showed in this study.
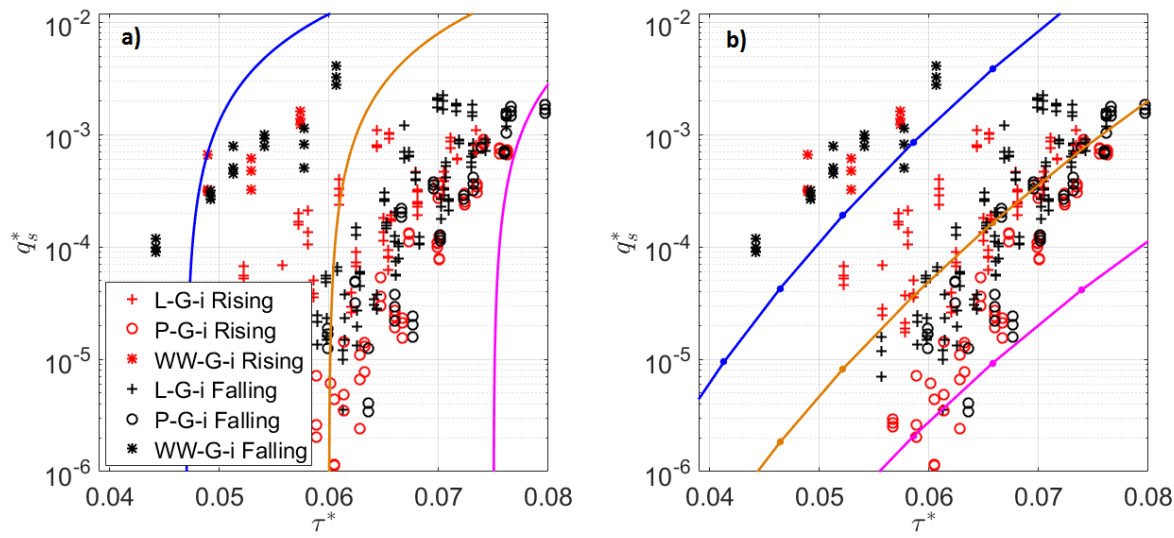

Fig. 4. $q_{s}{ }^{*}$ as a function of $\tau^{*}$. The lines approach data using (a) MPM or (b) Recking model $\left(\tau_{c r}{ }^{*}=\right.$ 0.047 for the blue line, $\tau_{c r}{ }^{*}=0.06$ for the orange line and $\tau_{c r}{ }^{*}=0.075$ for the magenta line).

\section{Conclusions and perspectives}

This paper shows the importance of taking into account bed arrangement when studying bedload dynamics. The bed arrangement impact on bedload was analysed using original laboratory experiments, which monitored bedload rates over different types of beds: loose, packed and water-worked. The latter was found to be significantly different according to the considered bed arrangement. Each type of bed had its own surface arrangement that was described with criteria deduced from the laser-scanner data analysis. The water-worked bed formed using only water power was the most organized and complex bed. Applying the same hydraulic conditions on each bed, we showed that gravel was transported more easily over a water-worked bed. Bedload rate was enhanced due to the presence of preferential pathways on the bed surface (areas of accelerated flows). The packed bed was the most imbricated. The transport of gravel was more difficult over this configuration due to higher bed stability. We were not able to express exhaustively bedload rate as a function of the bed 
surface parameters, but some correlations were exposed. Bedload rate was found to decrease with decreasing geometrical grain roughness, with presence of bed structures and preferential grain orientation and with increasing bed armoring degree. It also strongly increases with presence of large bedforms.

We tested some formulas such as the Meyer-Peter and Müller [13] or the Recking [14] formulas to examine their efficiency to predict bedload rate over beds approaching those of mountain rivers. Their predictive capabilities are generally poor for our specific cases, especially for packed beds. We suggest that bedload models include in their expressions a combination of bed surface parameters describing the bed arrangement of the studied bed.

The experiments were supported by Irstea, EDF (Electricité de France), the French Water Agency (AE-RMC) and the OSR research program (Rhône Sediment Observatory). The authors especially acknowledged A. Buffet for its technical support in conducting the laboratory experiments.

\section{References}

1. M. Church, M.A. Hassan, J.F. Wolcott, Stabilizing self-organized structures in gravelbed stream channels : field and experimental observations, WRR, 34 (1998)

2. M.A. Hassan, M. Church., Experiments on surface structure and partial sediment transport on a gravel bed, WRR, 36, 1885-1895 (2000)

3. J. Qin, S. Leung $\mathrm{Ng}$, Estimation of effective roughness for water-worked gravel surfaces, JHE, 138, 923-934 (2012)

4. A.C., Brayshaw, L.E. Frostick, I. Reid, The hydrodynamics of particle clusters and sediment entrainment in coarse alluvial channel, Sedimentology, 30, 137-143 (1983)

5. M.A. Hassan, R. Egozi, G. Parker, Experiments on the effect of hydrograph characteristics on vertical grain sorting in gravel bed rivers, WRR, 42 (2006)

6. L. Mao, The effect of hydrographs on bedload transport and bed sediment spatial arrangement, JGR: Earth Surf., 117 (2012)

7. I. Reid, L.E. Frostick, J.T. Layman, The incidence and nature of bedload transport during flood flows in coarse-grained alluvial channel, ESPL, 33-44 (1985)

8. A. Ockelford, H. Haynes, The impact of stress history on bed structure, ESPL, 38, 717727 (2013)

9. C. Berni, E. Perret, C. Camenen, Characteristic time of sediment transport decrease in static armour formation, submitted in Geomorphology (2018)

10. E. Perret, C. Berni, B. Camenen, A. Herrero, K. El kadi A., Transport of moderately sorted gravel at low bed shear stresses: the role of fine sediment infiltration, ESPL (in press)

11. J.R. Cooper, S.J. Tait, Water-worked gravel beds in laboratory flumes - a natural analogue?, ESPL, 34, 384-397 (2009)

12. E. Perret, C. Berni, A. Herrero, B. Camenen, A. Buffet, K. El kadi A., Laser scanning method to characterize bed arrangement and its influence on incipient motion of gravel sediments, River Flow (2016)

13. E., Meyer-Peter, R. Müller, Formulas for bedload transport, IAHR Congress (1948)

14. A. Recking, A comparison between flume and field bedload transport data and consequences for surface base bedload transport prediction, WRR, 46, 1-16 (2010) 